\title{
Total Anomalous Pulmonary Venous Connection in a 26 Year 0ld Adult- Echocardiographic Diagnosis and Surgical Correction
}

\author{
John Araujo ${ }^{1 *}$ and Rafael Meza Jimenez ${ }^{2}$ \\ ${ }^{1}$ Department of Clinic and Research, Cardiology Soma \& Meintegral Clinic-Medellin-Manizales, Colombia \\ ${ }^{2}$ Cardiovascular Surgeon in Pediatric and Adult Congenital Heart Diseases, Somer in Care Cardiovascular Center, Colombia
}

Submission: April 09, 2018; Published: July 13, 2018

*Corresponding author: John Araujo, Cardiologist-Echocardiologist in Pediatric and Adult Congenital Heart Diseases, Fellowship Integrated Unit of Congenital Heart Diseases of Adolescent and Adult Vall d'Hebron-Sant Pau, Vall d'Hebron University Hospital, Barcelona, Catalonia, Spain, Department of Clinic and Research Cardiology, Soma \& Meintegral Clinic, Postal code: 0954, Medellin-Manizales, Colombia, Tel: +5745768651; Email: johnjairoaraujo@gmail.com

Abstract

Total anomalous pulmonary venous connection is an infrequent congenital heart disease, Without surgical repair, mortality is greater than $50 \%$ in the first three months of life, and almost $90 \%$ at one year old. The incidence in adults is unknown; the literature reports anecdotal cases of late diagnosis. We present a case in adult 26 year old with total anomalous pulmonary venous connection intracardiac to coronary sinus and successful surgical repair.

Keywords: Total anomalous pulmonary venous connection; Pulmonary hypertension; Congenital heart disease

\section{Introduction}

Total anomalous pulmonary venous connection (TAPVC) is an infrequent congenital heart disease (CHD), accounting for $1-3 \%$ of all CHDs. It was first described in its partial form by Winslow in 1739 , and in its total form by Wilson in 1798 . The first systematizations based on embryology and anatomy were carried out by Nelly, Edwards and Helmholz in 1956 and by Darling in 1957. Muñoz Castellanos in 1968, and Van Praagh and Corsini in 1969, concluded that the anomaly results from a lack of incorporation of the pulmonary venous sinus into the left atrium (LA) [1]. Without surgical repair, mortality is greater than $50 \%$ in the first three months of life, and almost $90 \%$ at one year old. Death occurs earlier when the anomaly is obstructive (as evidenced by respiratory failure, severe heart failure and cyanosis), than when it is non-obstructive. The obstruction may occur at the interatrial septum (small defect), in an intrinsic narrowing of the anomalous venous collector, in a collector pressured by adjacent structures (esophageal hiatus, between the left pulmonary artery and the left bronchus), in the venous conduit due to the fact that it is normally constricted, and in the liver sinusoids. The development of pulmonary arterial hypertension (PAH) leads to a deterioration in functional class and death in survivors. Darling et al. classified TAPVC into four types: Type I: supracardiac (superior vena cava, the arch of the azygos vein, innominate vein and left superior vena cava); Type II: intracardiac (coronary sinus, right atrium or both); Type III: infracardiac (portal vein, hepatic veins, ductus venosus, gastric veins or inferior vena cava); and Type IV: mixed (occurring in two or more of the three levels described) [2]. In children, the order of frequency is supracardiac, $45 \%$; infracardiac, $25 \%$; intracardiac, $25 \%$; and mixed, $5 \%$ [3]. The incidence in adults is unknown; the literature reports anecdotal cases of late diagnosis, some with successful repair [4-7].

\section{Case Report}

A male 26 year old patient without prior hospitalizations, with a history of cyanosis beginning at age 10 . Three years ago he began experiencing dyspnea with moderate to mild exertion, and increased cyanosis, with an NYHA III functional class. On physical exam he had central cyanosis with $78 \%$ SO2 on room air, and $84 \%$ on oxygen at $3 \mathrm{~L} / \mathrm{min}$. He had a right parasternal hyperdynamic impulse; a normal first heart sound; a fixed intense, palpable, split second sound; and a IV/IV systolic midsternal murmur. Peripheral pulses were normal with hypotrophic extremities. Weight: 49kg, height: $161 \mathrm{~cm}$. Lab results: hemoglobin $(\mathrm{Hgb}) 19 \mathrm{gr} / \mathrm{dl}$; hematocrit (Hct) 57\%; platelets: 180,000, normal leukocytes. Chest x-ray: cardiomegaly, right heart chamber enlargement, dilated pulmonary trunk and branches, and pulmonary hyper 
flow. Electrocardiogram: sinus rhythm, QRS: $120^{\circ}$, right His bundle branch block, right heart chamber hypertrophy and signs of systolic overload. Transthoracic echocardiogram: intracardiac to coronary sinus TAPVC, $24 \mathrm{~mm}$ ostium secundum atrial septal defect (ASD) with right to left flow, dilation and hypertrophy of the right ventricle (RV), severe tricuspid insufficiency (TI vel max $4.2 \mathrm{~m} / \mathrm{s}$ ), and pulmonary hypertension with a calculated pulmonary systolic pressure of $82 \mathrm{mmHg}$ (equivalent to $2 / 3$ of the systemic pressure: $120 / 80 \mathrm{mmHg}$ ). The left ventricle (LV) was smaller with normal systolic function (Figure 1). Cardiac catheterization: pulmonary hypertension without coronary artery lesions (Table 1). Cardiac magnetic resonance confirmed the diagnosis and showed good left ventricular function, QP: 4.5; QS: 1. Surgical repair was carried out with coronary sinus (CS) un roofing and ASD closure with a patch, leaving a residual ASD gauged to be $4 \mathrm{~mm}$ (Figure 2). He had an adequate postoperative progress, with an SO2 of $94 \%$ without oxygen, Hgb $14 \mathrm{gr} / \mathrm{dl}$, and Hct $46 \%$. At follow up one year later he had no cardiovascular symptoms and was in functional class I. S02: 96\%. Echocardiogram: $4 \mathrm{~mm}$ ASD with left to right flow, and a pulmonary systolic pressure of $32 \mathrm{mmHg}$.

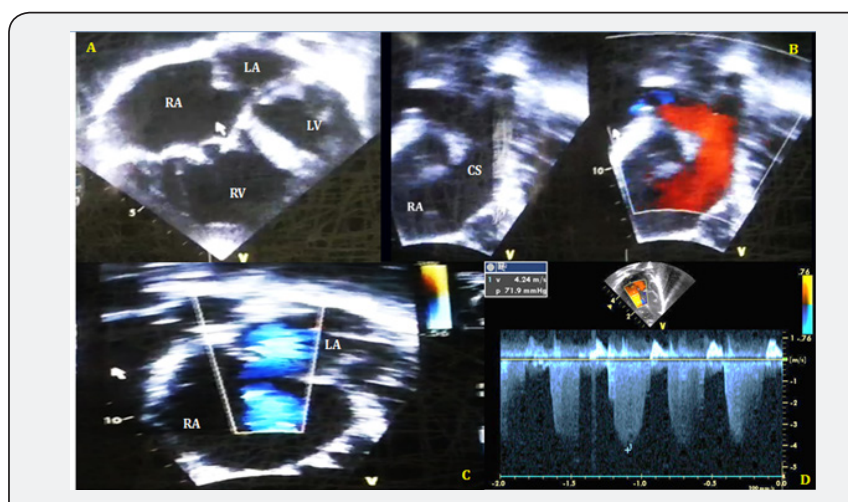

Figure 1: A: Apical four chambers view shows dilated $R V$ and RA, small or incomplete LA; B: Subcostal view shows total anomalous pulmonary venous connection to SC; C: Subcostal atrium view shows SDA flux right to left; D: Severe tricuspid insufficiency with high pulmonary systolic pressure. RA: Right Atrium; LA: Left Atrium; RV: Right Ventricle; LV: Left Ventricle; CS: Coronary Sinus

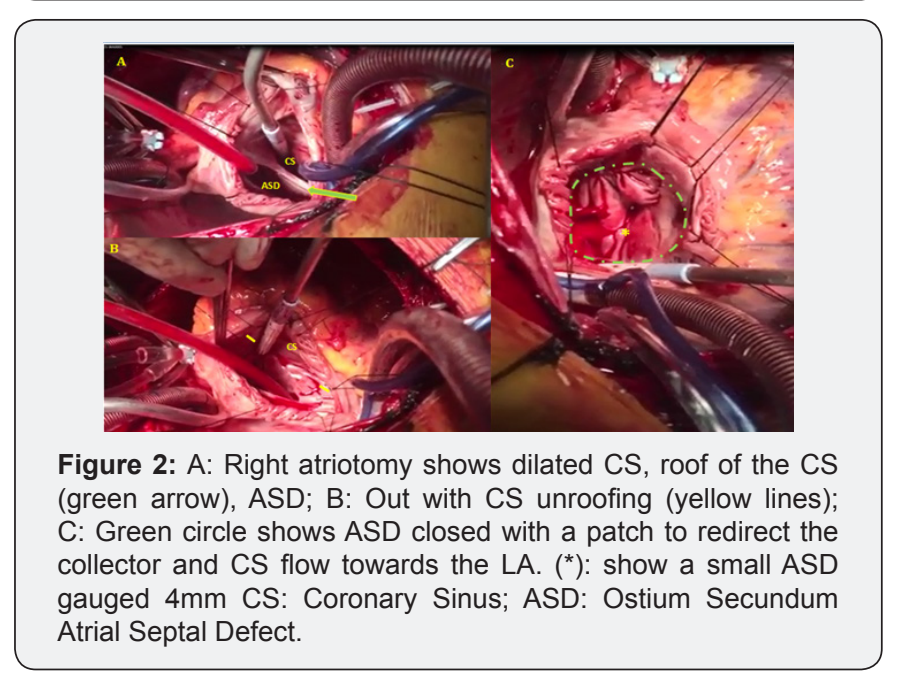

Table 1: Hemodynamic values cardiac catheterization.

\begin{tabular}{|c|c|c|}
\hline Parameter & Basal with IFO2 & Test with NO 20ppm \\
\hline IFO2 & $21 \%$ & $100 \%$ \\
\hline SBP mmhg & 125 & 118 \\
\hline DBP mmhg & 75 & 70 \\
\hline MSP mmhg & 91.7 & 86 \\
\hline SPAP mmhg & 110 & 95 \\
\hline DPAP mmhg & 75 & 52 \\
\hline MPAP mmhg & 84 & 66 \\
\hline SVR wu & 20.2 & 20.2 \\
\hline PVR wu & 10.7 & 6.5 \\
\hline PVR/SVR & 0.52 & 0.32 \\
\hline PCWP mmhg & 11 & 11 \\
\hline RAP mmhg & 17 & 14 \\
\hline CO lt $/$ min & 3.8 & 3.8 \\
\hline IC & 2.5 & 2.7 \\
\hline CF min & 75 & 77 \\
\hline
\end{tabular}

Weight $49 \mathrm{~kg}$, Height $161 \mathrm{~cm}$, BS $1.5 \mathrm{~m}^{2}$

SBP: Systolic Blood Pressure; DBP: Diastolic Blood Presure; MSP: Medium Systemic Pressure; SPAP: Systolic Pulmonary Artery Pressure; DPAP: Diastolic Pulmonary Artery Pressure; MPAP: Medium Pulmonary Artery Pressure; SVR: Systemic Vascular Resistance; PVR: Pulmonary Vascular Resistance; VPR/SVR: Index; PCWP: Pulmonary Capillary Wedge Pressure; RA: Right Atrium Pressure; CO: Cardiac Output; IC: Index Cardiac; CF: Cardiac Frequency; BS: Body Surface: ON: Nitric Oxide. IFO2: Inspired Fraction Oxygen: WU: Wood Units

\section{Discussion}

In all types of TAPVC, the obligatory defect is undoubtedly an ASD, which allows blood from the pulmonary veins to reach the left cardiac chambers and systemic circulation. When the ASD is large, the pressures in both atria are equal; the amount of atrial flow depends on the distensibility of the ventricles and the resistance to ventricular emptying. When the pulmonary vascular resistance (PVR) diminishes in the first weeks of life, right ventricular distensibility, and consequently pulmonary flow, increases. Under these conditions there is only a slight decrease in peripheral saturation, with minimal cyanosis, which may not be noticed. The progression will be similar to that of an isolated ASD. The case presented corresponds to Darling's type II, with a good ASD which allowed survival into adulthood. In expert hands, the echocardiogram is diagnostic in almost $100 \%$ of cases [8]. An echocardiographic study should show: the absence of connected pulmonary veins in the LA; identification of the collector (venous convergence and pulmonary veins); identification of the exact connection site; evaluation of the presence and degree of obstruction; associated lesions; and the degree of hemodynamic impact. For the current case, the diagnostic keys are:

1. Incomplete LA (the venous portion is lacking)

2. Marked dilation of the right chambers with signs of volume overload 
3. Great dilation of the CS (in the absence of a persistent left vena cava)

4. Colour Doppler image of the pulmonary venous collector draining to the coronary sinus ("whale's tail" appearance) (Figure 3)

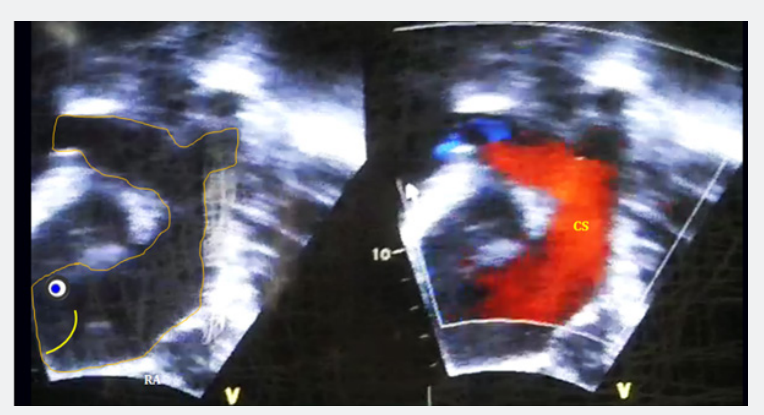

Figure 3: Subcostal view shows the classic image "whale's tail appearance", the coronary sinus is verticalized and filled completely by the flow of the pulmonary veins. CS: Coronary Sinus.

5. A smaller LV

6. ASD and PAH

Persistent increased pulmonary flow leads to PAH, endothelial hyperplasia and hypertrophy of the medial layer of the arterioles, and increased PVR. Secondary to this, there is a progressive decrease in pulmonary flow and increased right to left flow through the ASD with the onset of, or increased cyanosis, RV pressure overload and heart failure. In the case presented here, the low SO2 at diagnosis with a high Hgb and Hct as an adaptive consequence of chronic hypoxemia, associated with clinical and echocardiographic signs of PAH made it necessary to perform a cardiac catheterization to measure the pulmonary pressure and PVR. It is accepted that a CHD may be repaired if the following criteria are met: under baseline conditions, if the PVR is $\leq 6 \mathrm{WU} /$ $\mathrm{m} 2$; and the PVR/SVR ratio is $\leq 0.3$. In the event that the PVR is $\geq 6-9 \mathrm{WU} / \mathrm{m} 2$ and the PVR/SVR ratio is $0.3-0.5$, a pharmacological challenge with nitric oxide is required to determine pulmonary vascular reactivity. The challenge is considered to be positive if the PVR or MPAP decrease by $20 \%$, the PVR/SVR ratio decreases by $20 \%$, or the PVR is $\leq 6 \mathrm{WU} / \mathrm{m} 2$ and the PVR/SVR ratio is $\leq 0.3$ [9]. In congruence with the expert recommendations, we decided to perform the surgical correction.

Three surgical repair techniques have been described [10]:

1. The classic Van Praagh technique, in which the superolateral wall of the CS next to the LA is opened, leaving an unroofed CS, and then the outlet to the right atrium (RA) is closed with direct sutures, and the ASD is also closed.

2. A modification of the prior technique, in which the CS wall is also opened, but its outlet to the RA is tunneled towards the LA through an enlarged ASD (if it was previously restrictive) using a heterologous patch (pericardium, polytetrafluoroethylene, dacron) or an autologous pericardial patch treated with glutaraldehyde.

3. Sectioning of the muscular bridge which separates the collector outlet opening in the CS and the ASD in order to create a large ASD which is then closed with a patch to redirect the collector and CS flow towards the LA.

We opted by number 3 technique repair, leaving, in addition, a small ASD gauged to be $4 \mathrm{~mm}$ as an escape route in the event of a pulmonary hypertensive crisis immediately after surgery (a very common complication in cases where PAH has been diagnosed). The values found on cardiac catheterization after the pulmonary vasodilator test were accepted for closure (MPAP, PVR/SVR ratio decreased by more than 20\%); the absolute PVR was not below $6 \mathrm{WU}$, but it did decrease by more than $20 \%$, and the PVR/SVR ratio was borderline (0.32). Without surgical repair, the progression to irreversible $\mathrm{PAH}$ (Eisenmenger situation), functional class deterioration and death would have been high. When the PVR is between 6-8WU, the surgical repair cases can be individualized, just as we decided with this patient. All of these patients must receive strict follow-up due to the high possibility of developing progressive postoperative $\mathrm{PAH}$, even years after repair. Ten percent of adult cases develop supraventricular arrhythmias (flutter). In our case, the patient still has a sinus rhythm. However, he will need to be followed for life.

\section{Conclusion}

TAPVC is a rare CHD, with a high mortality rate in childhood. Cases of survival into adulthood are infrequently reported in the medical literature. Unquestionably, the presence of a large ASD is key in this patients' survival. In adults, a cardiac catheterization is mandatory in order to make a correct surgical decision. Follow-up must be for life due to the potential risk of developing postoperative $\mathrm{PAH}$ and arrhythmias. In expert hands, the echocardiogram is diagnostic in almost $100 \%$ of cases.

\section{References}

1. Muñoz L, Kuri M, Sánchez C, Espínola N (2008) Conexión anómala de venas pulmonares. Correlación Anatomoembriológica. Arch Cardiol Méx 78(3): 47-54.

2. Patiño E, Calderón J, Buendía A, Attie F (2013) Conexión anómala total de venas pulmonares. In: Attie F, Calderon J, Zabal C, Buendia A, (Eds.), Cardiología Pediátrica. (2 ${ }^{\text {nd }}$ edn), DF: Editorial Médica Panamericana, México, pp. 93-102.

3. Kanter KR (2006) Surgical repair of total anomalous pulmonary venous connection. Semin Thorac Cardiovasc Surg Pediatr Card Surg Annu 9(1): 40-44.

4. Wetzel U, Scholtz W, Bogunovic N, Körfer J, Haas NA, et al. (2010) Successful correction of a total anomalous venous connection in a 63-year-old male-case report and review of the literatura Congenit. Heart Dis 5: 470-475.

5. Hisagi M, Higuchi K, Koseni K, Inaba H (2006) Total anomalous pulmonary venous return in an adult. Asian Cardiovasc Thorac Ann 14(2): e27-e29. 
6. Ashoush R, Jebara VA, el Rassi I, Badawi G, Sarkis A, et al. (1993) Total anomalous pulmonary venous connection in adults-a surgical review. J Med Liban 41(4): 230-235.

7. Feng Q Wu S, Yu G (2010) Surgical treatment of a 56-year-old woman with an intracardiac type of total anomalous pulmonary venous connection. Thorac Cardiovasc Surg 58(3): 175-176.

8. Zhang Z, Zhang L, Xie F, Wang B, Sun Z, et al. (2016) Echocardiographic diagnosis of anomalous pulmonary venous connections experience of 84 cases from 1 medical center. Medicine 95(44): e5389.
9. Calderón J, Sandoval J, Beltrán M (2015) Hipertensión pulmonar asociada a cardiopatías congénitas y síndrome de Eisenmenger. Arch Cardiol Mex 85(1): 32-49.

10. Aroca A, Montserrat L, López-Ortego P, González A, Villagrá F (2014) Drenaje venosa pulmonar anómalo total. Técnicas y resultados. Cir Cardiov 21(2): 90-96. 